\title{
Surface Effects on the Scatter of SH-Wave by a Shallow Buried the Elliptical Hole
}

\author{
Z. Y. Ou*, Y. Q. Sun \\ School of Science, Lanzhou University of Technology, Gansu, Lanzhou, China \\ Email: *zhiyingou@163.com
}

How to cite this paper: Ou, Z.Y. and Sun, Y.Q. (2019) Surface Effects on the Scatter of SH-Wave by a Shallow Buried the Elliptical Hole. Open Journal of Applied Sciences, 9, 480-491.

https://doi.org/10.4236/ojapps.2019.96038

Received: May 22, 2019

Accepted: June 24, 2019

Published: June 27, 2019

Copyright (อ 2019 by author(s) and Scientific Research Publishing Inc. This work is licensed under the Creative Commons Attribution International License (CC BY 4.0).

http://creativecommons.org/licenses/by/4.0/

\begin{abstract}
The methods of complex function, multi-polar coordinate system, and conformal mapping are used to solve dynamic stress concentration factor. The surface elasticity theory is applied to obtain the stress boundary conditions on the surface. The effects of frequency and the ration of the major and minor axis of the ellipse on the dynamic stress concentration factor around the elliptical nano-hole are discussed in detail. When the size of elliptical hole shrinks to nanometers, the numerical results show that the surface effect has a significant effect on the scattering of SH-wave.
\end{abstract}

\section{Keywords}

Conformal Mapping, Surface Effect, Dynamic Stress Concentration Factor, Elliptical Hole, SH-Wave, Complex Function

\section{Introduction}

The scattering of elastic waves by microstructures in solids remains a fundamental issue both in theoretical researches and in engineering applications such as nondestructive detection and prediction of the overall dynamic properties of composites. Many works have been carried out on this subject based on the classical elastodynamics theory in literature [1]. The diffraction of elastic waves by a single inhomogeneity embedded in an elastic medium, as well as the dynamic stress concentration around the inhomogeneity, has been comprehensively reviewed by Pao and Mow [1]. These researches are mainly on the holes and inclusions of constant curvature in the whole space. Liu, et al. [2] [3] [4] [5] used the complex variable function and the multi-polar coordinate methods to introduce the conformal mapping to analyze the scattering problems of the variable curvature holes. Shi, et al. [6] [7] [8] used the mirror method to extend the whole space problem to half space. Lee, et al. [9] discussed circular tunnels in half space 
and obtained analytical solutions. Manoonan, et al. [10] used the weighted parameter method to solve the elastic half-space surface displacement of circular, elliptical and square inclusions by SH-wave. Yuan, et al. [11] studied the scattering and vibration of $\mathrm{SH}$-wave by shallow cylindrical holes. Using a variety of different methods, many problems in life have been theoretically solved [12] [13] [14] [15] [16].

With the rapid development of micro-nano-components, there is an increasing demand for understanding the mechanical behavior of small-sized materials and structures, which often differ distinctly from their macroscopic counterparts. As the volume of the object decreases, the ratio of surface area to volume increases, and the surface effect is significant, thus exhibiting mechanical behavior different from the macroscopic case. For example, geckos can walk freely on vertical walls and mosquitoes walk on the water. Based on Gurtin's surface elasticity theory [17] [18], Sharma, et al. [19] studied the size dependence of the elastic field around the nano-cylindrical and nano-sphere inclusions in the whole space. Using the wave function expansion method, Wang, et al. [20] [21] discussed the diffraction of plane compressional wave (P-wave) in nano-hole. Shen, et al. [22] discussed the influence of surface effects on the stress field around nano-inclusions. Ou, et al. [23] [24] [25] discussed the mechanical behaviors of inclusions and holes subjected to uniform loads at nano-scale.

In the present work, we discuss the scattering of plane SH-wave by an elliptical hole in elastic half-space at nano-scale. Based on the classical boundary conditions, the conformal mapping method is used to establish the boundary model and the stress concentration problem is analyzed by using the methods of wave function expansion and complex variable function.

\section{Basic Equations}

In the surface elasticity theory, a surface is regarded as negligibly thin membranes adhere to the bulk without slipping and it has material constants different from those of the bulk. The equilibrium and constitutive equations in the bulk of the solid read

$$
\begin{gathered}
\tau_{i j, j}=\rho \frac{\partial^{2} u_{i}}{\partial t^{2}} \\
\tau_{i j}=2 \mu\left(\varepsilon_{i j}+\frac{v}{1-2 v} \varepsilon_{k k} \delta_{i j}\right),
\end{gathered}
$$

where $\rho$ is the mass density of the material, $t$ is the time. $\mu$ and $v$ are respectively shear modulus and Poisson's ration, $\tau_{i j}$ and $\varepsilon_{i j}$ are the stress tensor and strain tensor in the bulk material. The strain tensor is related to the displacement vector $u_{i}$ by

$$
\varepsilon_{i j}=\frac{1}{2}\left(u_{i, j}+u_{j, i}\right) .
$$

The equilibrium equations on the surface are expressed as [18] 


$$
t_{\alpha}+\tau_{\beta \alpha, \beta}^{s}=0, \quad \tau_{i j} n_{i} n_{j}=\tau_{\alpha \beta}^{s} \kappa_{\alpha \beta} .
$$

where $t_{\alpha}$ is the negative of the tangential component of the traction $t_{i}=\tau_{i j} n_{j}$ in the $x_{\alpha}$ direction, and $n_{i}$ is the normal vector of the surface, $\kappa_{\alpha \beta}$ denotes the curvature of the surface. The surface stresses are given for anisotropic surface by [15] [19]

$$
\tau_{\alpha \beta}^{s}=2 \mu^{s} \varepsilon_{\alpha \beta}+\lambda^{s} \varepsilon_{\gamma \gamma} \delta_{\alpha \beta} .
$$

where $\varepsilon_{\alpha \beta}$ is the second-rank tensor of surface strain, $\delta_{\alpha \beta}$ is the Kronecker delta, and $\mu^{s}$ and $\lambda^{s}$ are surface elastic constants.

For a circular hole of the radius $r=a$, according to the Equation (4) and Equation (5), we find

$$
\tau_{r z}=-\frac{1}{a} \frac{\partial \tau_{\theta z}^{s}}{\partial \theta}, \quad \tau_{\theta z}^{s}=2 \mu^{s} \varepsilon_{\theta z} .
$$

The boundary condition of stress around the circular hole can be obtained from Equation (6) as follows

$$
\tau_{r z}=-s \frac{\partial \tau_{\theta z}}{\partial \theta},
$$

where

$$
s=\frac{\mu^{s}}{\mu a} .
$$

with $s$ being a dimensionless parameter reflecting the effect of the surface/interface at nano-scale. It is seen from Equation (8) that for a macroscopic hole with a big radius, $s \rightarrow 0$, therefore the surface/interface effect can be neglected. However, when the radius of the hole shrinks to nano-scale, $s$ becomes noticeable and the surface effect should be considered in the analysis [18] [19].

\section{Theoretical Model and Analysis}

Consider an elliptical nano-hole embedded in an elastic half-space, as shown in Figure 1. It is assumed that the medium is elastic, uniform, isotropic and linear. The long semi-axis of the elliptical hole is denoted by a, and the short semi-axis is denoted by $b$.

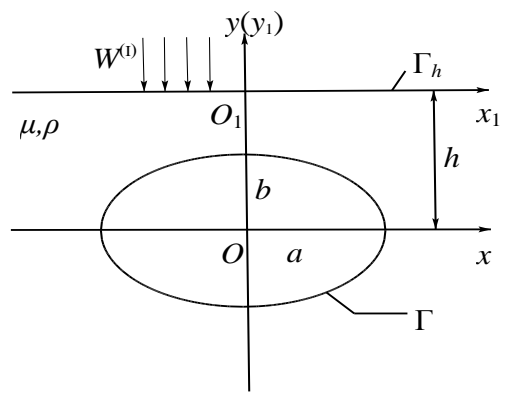

Figure 1. Scattering of SH-wave by an elliptical hole embedded in the half space. 
If there is no hole and only incident plane wave exists in the half-space, the incident wave can be represented in the coordinate system as [5]

$$
w^{(\mathrm{I})}=w_{0} \exp \left[-\mathrm{i}\left(k y_{1}+\omega t\right)\right] .
$$

where $w_{0}$ represents the amplitude of the incident wave, $i$ is the unit of imaginary number, $k=\omega / c_{s}$ is the number of the incident wave, $c_{s}=\sqrt{\mu / \rho}$ represents the shear wave velocity of the medium, $\rho$ and $\mu$ are the mass density and shear modulus of the medium respectively.

For the steady-state problem, the time-factor $\mathrm{e}^{-\mathrm{i} \omega t}$ is ignored; the wave displacement fulfills the following Helmholtz equation in the medium

$$
\frac{\partial^{2} w}{\partial x^{2}}+\frac{\partial^{2} w}{\partial y^{2}}+k^{2} w=0
$$

The relationships between stress and displacement are

$$
\tau_{x z}=\mu \frac{\partial w}{\partial x}, \quad \tau_{y z}=\frac{\partial w}{\partial y} .
$$

Introducing the complex plane $(z, \bar{z})$, where the complex variable $z=x+i y$, $\bar{z}=x-i y$. The Equation (10) can be expressed as follows

$$
\frac{\partial^{2} w}{\partial z \partial \bar{z}}+\frac{k^{2}}{4} w=0 .
$$

The incident wave passes through the half space to the boundary of the elliptical hole, which will generate scattering wave at the boundary of the hole. Using the virtual source method, the scattering wave function that satisfies the stress freeness at the horizontal boundary is expressed as [5]

$$
w^{(\mathrm{s})}=\sum_{n=-\infty}^{\infty} A_{n}\left[H_{n}^{(1)}(k|z|)\left(\frac{z}{|z|}\right)^{n}+H_{n}^{(1)}(k|z-2 h \mathrm{i}|)\left(\frac{z-2 h \mathrm{i}}{|z-2 h \mathrm{i}|}\right)^{-n}\right]
$$

where $A_{n}$ are the unknown coefficients and determined by the boundary conditions, $H_{n}^{(1)}(\cdot)$ is the first kind Hankel function of the $n$-th order.

Introducing conformal mapping function $z=\omega(\eta)=R(\eta+m / \eta)$ from elliptical hole to unit circular hole $(r=1)$, the boundary $\Gamma$ of the elliptical hole is mapped to the unit circle boundary $\Gamma_{v}$ as shown in Figure 2.

here $R=\frac{a+b}{2}, m=\frac{a-b}{a+b}, \eta=\xi+\mathrm{i} \zeta, \bar{\eta}=\xi-\mathrm{i} \zeta$.

Substituting the conformal mapping function $z=\omega(\eta)$ into the Equation (9), Equation (11), Equation (12) and Equation (13), we can obtain

$$
\begin{gathered}
w^{(\mathrm{I})}=w_{0} \exp \left\{-\frac{k}{2}[\omega(\eta)-\mathrm{i} h]+\frac{k}{2}[\overline{\omega(\eta)}+\mathrm{i} h]\right\} \\
\frac{1}{\omega^{\prime}(\eta) \overline{\omega^{\prime}(\eta)}} \frac{\partial^{2} w}{\partial \eta \partial \bar{\eta}}+\frac{k^{2}}{4} w=0, \\
\tau_{r z}=\frac{\mu}{\left|\omega^{\prime}(\eta)\right|}\left(\eta \frac{\partial w}{\partial \eta}+\bar{\eta} \frac{\partial w}{\partial \bar{\eta}}\right),
\end{gathered}
$$




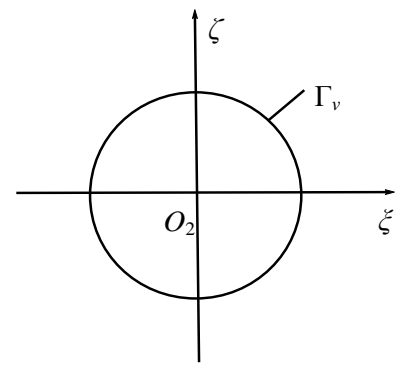

Figure 2. Image plane after conformal transformation.

$$
\begin{gathered}
\tau_{\theta z}=\frac{\mathrm{i} \mu}{\left|\omega^{\prime}(\eta)\right|}\left(\eta \frac{\partial w}{\partial \eta}-\bar{\eta} \frac{\partial w}{\partial \bar{\eta}}\right) \\
w^{(s)}=\sum_{n=-\infty}^{\infty} A_{n}\left\{H_{n}^{(1)}[k|\omega(\eta)|]\left[\frac{\omega(\eta)}{|\omega(\eta)|}\right]^{n}+\varphi\right\}
\end{gathered}
$$

where

$$
\varphi=H_{n}^{(1)}[k|\omega(\eta)-2 h \mathrm{i}|]\left[\frac{\omega(\eta)-2 h \mathrm{i}}{|\omega(\eta)-2 h \mathrm{i}|}\right]^{-n}
$$

In order to improve the convergence speed and calculation accuracy, introducing Graf addition formula [26]

$$
\varphi=\psi^{(1)}
$$

where

$$
\psi^{(1)}=\sum_{t=-\infty}^{\infty} \mathrm{e}^{\mathrm{i}(t-n) \pi / 2} H_{t-n}^{(1)}(2 k h) J_{t}[k|\omega(\eta)|]\left[\frac{\omega(\eta)}{|\omega(\eta)|}\right]^{-t},
$$

where $J_{t}(\cdot)$ is the first kind Bessel function of the $n$-th order. Substituting Equation (20) into Equation (18), we can obtain

$$
w^{(s)}=\sum_{n=-\infty}^{\infty} A_{n}\left\{H_{n}^{(1)}[k|\omega(\eta)|]\left[\frac{\omega(\eta)}{|\omega(\eta)|}\right]^{n}+\psi^{(1)}\right\}
$$

\section{Problem Solving}

Substituting Equation (14) into Equation (16) and Equation (17), we can obtain stress expressions of the incident wave as

$$
\begin{aligned}
\tau_{r z}^{(\mathrm{I})} & =-\mu k w_{0} \exp \left\{-\frac{k}{2}[\omega(\eta)-\mathrm{i} h]+\frac{k}{2}[\overline{\omega(\eta)}+\mathrm{i} h]\right\} \frac{\mathrm{i} \operatorname{Im}\left[\eta \omega^{\prime}(\eta)\right]}{\left|\omega^{\prime}(\eta)\right|} \\
\tau_{\theta z}^{(\mathrm{I})} & =-\mathrm{i} \mu k w_{0} \exp \left\{-\frac{k}{2}[\omega(\eta)-\mathrm{i} h]+\frac{k}{2}[\overline{\omega(\eta)}+\mathrm{i} h]\right\} \frac{\operatorname{Re}\left[\eta \omega^{\prime}(\eta)\right]}{\left|\omega^{\prime}(\eta)\right|} .
\end{aligned}
$$

and substituting Equation (22) into Equation (16) and Equation (17), stress expressions of the scattering wave can be expressed as 


$$
\begin{aligned}
\tau_{r z}^{(\mathrm{s})}= & \frac{\mu k w_{0}}{2} \sum_{n=-\infty}^{\infty} A_{n}\left\{H_{n-1}^{(1)}[k|\omega(\eta)|]\left[\frac{\omega(\eta)}{|\omega(\eta)|}\right]^{n-1} \frac{\eta \omega^{\prime}(\eta)}{\left|\omega^{\prime}(\eta)\right|}-\psi^{(2)} \frac{\eta \omega^{\prime}(\eta)}{\left|\omega^{\prime}(\eta)\right|}\right. \\
& \left.-H_{n+1}^{(1)}[k|\omega(\eta)|]\left[\frac{\omega(\eta)}{|\omega(\eta)|}\right]^{n+1} \frac{\bar{\eta} \overline{\omega^{\prime}(\eta)}}{\left|\omega^{\prime}(\eta)\right|}+\psi^{(3)} \frac{\bar{\eta} \overline{\omega^{\prime}(\eta)}}{\left|\omega^{\prime}(\eta)\right|}\right\} \\
\tau_{\theta z}^{(\mathrm{s})}= & \frac{\mathrm{i} \mu k w_{0}}{2} \sum_{n=-\infty}^{\infty} A_{n}\left\{H_{n-1}^{(1)}[k|\omega(\eta)|]\left[\frac{\omega(\eta)}{|\omega(\eta)|}\right]^{n-1} \frac{\eta \omega^{\prime}(\eta)}{\left|\omega^{\prime}(\eta)\right|}-\psi^{(2)} \frac{\eta \omega^{\prime}(\eta)}{\left|\omega^{\prime}(\eta)\right|}\right. \\
& \left.+H_{n+1}^{(1)}[k|\omega(\eta)|]\left[\frac{\omega(\eta)}{|\omega(\eta)|}\right]^{n+1} \frac{\bar{\eta} \overline{\omega^{\prime}(\eta)}}{\left|\omega^{\prime}(\eta)\right|}-\psi^{(3)} \frac{\bar{\eta} \overline{\omega^{\prime}(\eta)}}{\left|\omega^{\prime}(\eta)\right|}\right\}
\end{aligned}
$$

where

$$
\begin{aligned}
& \psi^{(2)}=\sum_{t=-\infty}^{\infty} \mathrm{e}^{\mathrm{i}(t-n-1) \pi / 2} H_{t-n-1}^{(1)}(2 k h) J_{t}[k|\omega(\eta)|]\left[\frac{\omega(\eta)}{|\omega(\eta)|}\right]^{-t} \\
& \psi^{(3)}=\sum_{t=-\infty}^{\infty} \mathrm{e}^{\mathrm{i}(t-n+1) \pi / 2} H_{t-n+1}^{(1)}(2 k h) J_{t}[k|\omega(\eta)|]\left[\frac{\omega(\eta)}{|\omega(\eta)|}\right]^{-t}
\end{aligned}
$$

where $\tau_{r z}^{(\mathrm{I})}$ and $\tau_{r z}^{(\mathrm{s})}$ are the radial stress, $\tau_{\theta z}^{(\mathrm{I})}$ and $\tau_{\theta z}^{(\mathrm{s})}$ are tangential stress, $\mathrm{Re}$ is the real part and Im is the imaginary part of a complex variable.

The total stress and total displacement are expressed as

$$
\begin{gathered}
\tau_{r z}=\tau_{r z}^{(\mathrm{I})}+\tau_{r z}^{(\mathrm{s})}, \\
\tau_{\theta z}=\tau_{\theta z}^{(\mathrm{I})}+\tau_{\theta z}^{(\mathrm{s})} \\
w=w^{(\mathrm{I})}+w^{(\mathrm{s})}
\end{gathered}
$$

The boundary conditions of the boundary of the half space and the unit circle hole respectively are

$$
\Gamma_{v}: \tau_{r z}(r, \theta)=-s \frac{\partial \tau_{\theta z}}{\partial \theta}, \Gamma_{h}: \tau_{y z}(x, h)=0
$$

As shown in Equation (13), the scattering wave will be determined if the unknown coefficients $A_{n}$ are solved. Substituting Equation (23), Equation (24), Equation (25) and Equation (26) into Equation (32), we find

$$
\sum_{n=-\infty}^{\infty} A_{n} \varepsilon_{n}+\varepsilon=0,
$$

where the terms $\varepsilon_{n}$ and $\varepsilon$ are presented in Appendix A. It is evident that Equation (33) is a series of equations related to $\theta$ though there are still unknown coefficients. Multiplying both sides of Equation (33) with $\mathrm{e}^{-\mathrm{im} \theta}$ and integrating between the interval $(-\pi, \pi)$, we can obtain

$$
\sum_{n=-\infty}^{\infty} \varepsilon_{n}^{m} A_{n}+\varepsilon^{m}=0
$$

where $\varepsilon_{n}^{m}=\frac{1}{2 \pi} \int_{-\pi}^{\pi} \varepsilon_{n} \mathrm{e}^{-\mathrm{i} m \theta} \mathrm{d} \theta, \quad \varepsilon^{m}=\frac{1}{2 \pi} \int_{-\pi}^{\pi} \varepsilon \mathrm{e}^{-\mathrm{i} m \theta} \mathrm{d} \theta$. 
A set of infinite algebraic equations with respect to the unknown constants $A_{n}$ will be obtained from Equation (34). It should be noted that it is mathematically convenient to truncate the infinite matrix in Equation (34) with $n=m$ during numerical calculation.

\section{Problem Results and Analysis}

Elastic-wave-induced dynamic stress concentration factor (DSCF) is an important parameter for engineering application. The SH-wave-induced DSCF is calculated as

$$
\operatorname{DSCF}=\left|\frac{\tau_{\theta z}}{\tau_{0}}\right| .
$$

where $\tau_{0}=\mu k w_{0}$ is the maximum amplitude of the incident stress.

To validate the present approach, a comparison with Liu's conclusions is shown in Figure 3. It can be seen that the present results on DSCF around a circular hole in the half-space with a surface parameter $s=0$ are accordant with Liu's conclusions [4]. Figure 4 shows the distributions of DSCF about the angle $\theta$ with different values of the surface parameter $s$. With the increase of $s$, the DSCF continuously decreases. It is seen that surface energy significantly affects DSCF around the hole.

We discuss the influences of surface effects, incident wave frequencies and the ration of the major and minor axis of the ellipse on the dynamic stress concentration factor near an elliptical hole now.

Figure 5 shows the distributions of DSCF about the angle $\theta$, when $h / a=1.5, b / a=0.6$ and $k a=\pi$. The results indicate that DSCF is symmetric about the y-axis. With the increasing of $s$, the DSCF continuously decreases around $\theta / \pi<0.15$ or $\theta / \pi>0.85$. When $s=2$, the DSCF in the ranges of $0.35<\theta / \pi<0.65$ is greater than the cases of $s=0.1$ and $s=0.5$. When $\theta / \pi=0.5$, the DSCF is zero.

Next, we discuss the distributions of DSCF around the elliptical hole at different frequencies, when $a / b=0.6$. It is seen that when the surface/interface effect is taken into account, the dynamic stress depends not only on $s$ but also on the frequencies. If $k \rightarrow 0$ and keep $\tau_{0}$ as a constant, then the incident $\mathrm{SH}$-wave represents a static biaxial loading [1].

For a high-frequency incident wave with $k a=\pi$. Figure 6 displays the distributions of DSCF around the elliptical hole for different interface parameter $s$. In this case, the DSCF is symmetric about the $y$-axis. With increasing $s$, the DSCF declines continuously in the angular range of $0.15<\theta / \pi<0.95$. For a low-frequency incident wave with $k a=0.2$, the distributions of DSCF around the elliptical hole for different values $s$ are plotted in Figure 7. It can be seen that DSCF decreases in the angular range of $0.05<\theta / \pi<0.95$ increasing $s$ but slowly increases elsewhere. Comparing the high frequency and low frequency, we find that DSCF shows a decreasing trend as the frequency increases. 


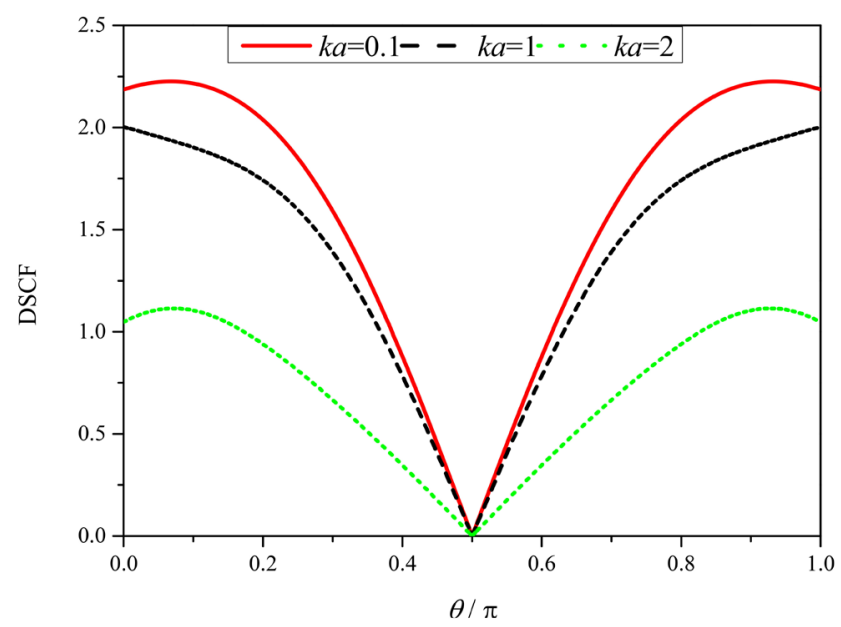

Figure 3. The distribution of DSCF near a circular hole for different wave number with $h / a=1.5$.

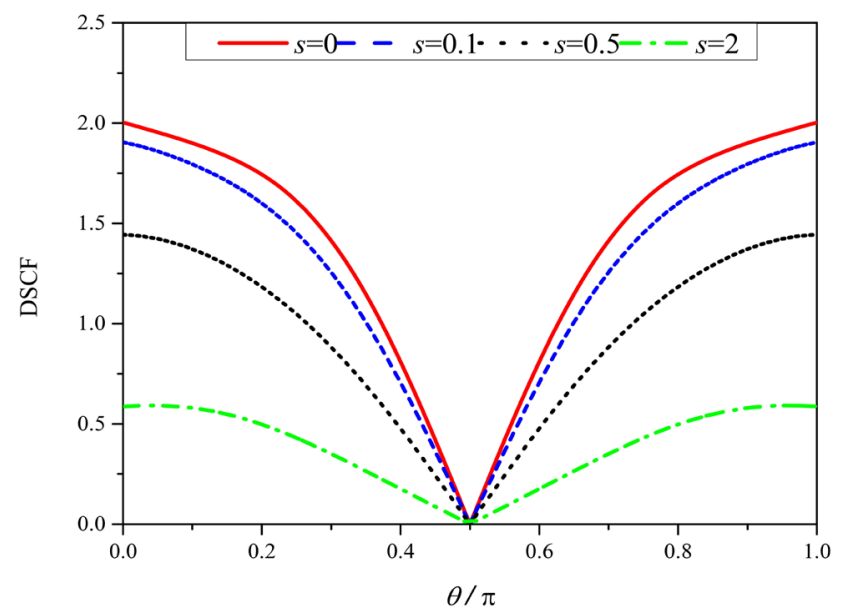

Figure 4. Effect of interface parameter $s$ on DSCF near a circular hole for $k a=1, h / a=1.5$.

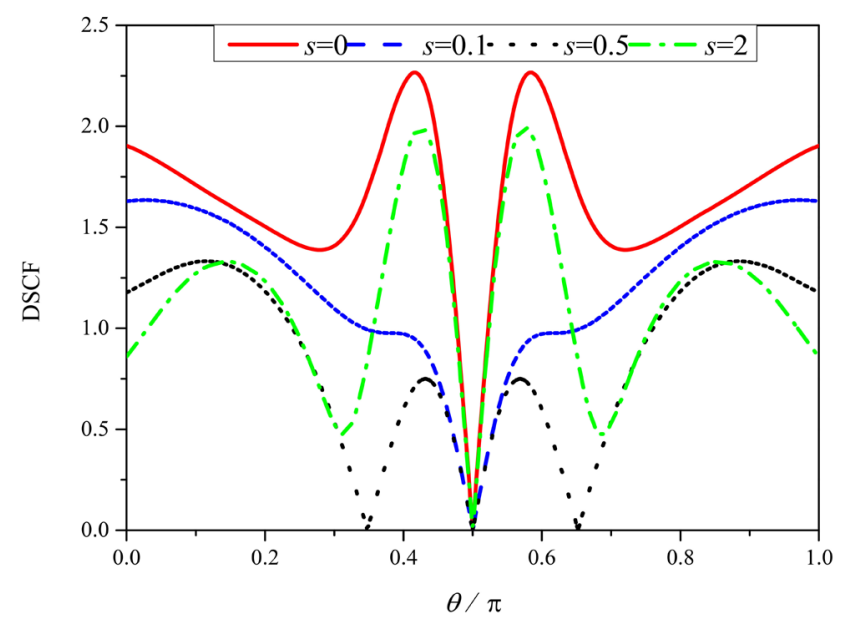

Figure 5. Effect of interface parameter $s$ on DSCF near an elliptical hole for $k a=\pi, \quad b / a=0.6$. 


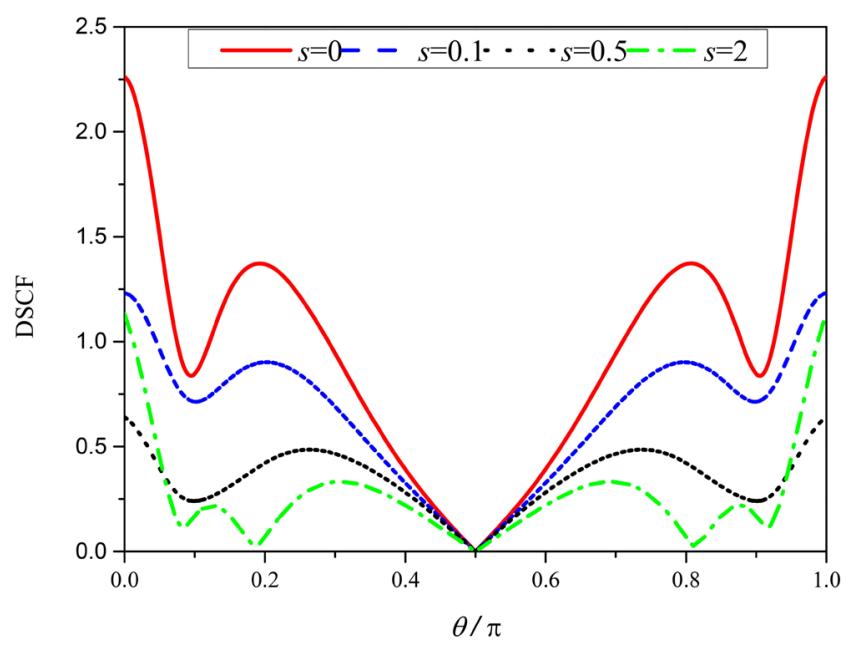

Figure 6. Effect of interface parameter $s$ on DSCF near an elliptical hole for $k a=\pi, a / b=0.6$.

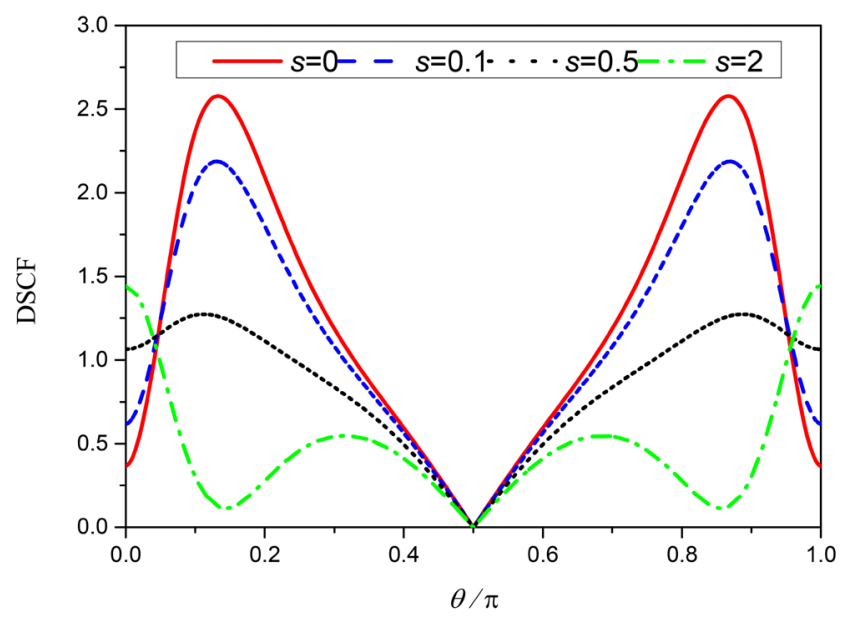

Figure 7. Effect of interface parameter $s$ on DSCF near an elliptical hole for $k a=0.2, a / b=0.6$.

\section{Conclusion}

In this paper, based on the theory of surface/interface elasticity, we focused on the observation that dynamic stress concentration factor of the elliptical hole. We used conformal mapping to transform an elliptical hole into a unit circular hole. Therefore, it simplifies the process of processing elliptical boundary conditions. The numerical solutions of the elastic fields induced by SH-wave near an elliptical nano-hole are obtained. The influence of surface/interface effect on the DSCF is discussed. We get the conclusion that DSCF around the hole is not only related to interface effects, but also related to frequencies.

\section{Acknowledgements}

The supports from the National Natural Science Foundation (Grant No. 11362009 and No. 11862014) are acknowledged. 


\section{Conflicts of Interest}

The authors declare no conflicts of interest regarding the publication of this paper.

\section{References}

[1] Mow, C.C. and Pao, Y.H. (1973) The Diffraction of Elastic Waves and Dynamic Stress Concentration. Journal of Applied Physics, 40, 872-1500. https://doi.org/10.1115/1.3423178

[2] Wang, G.Q. and Liu, D.K. (2003) Dynamic Analysis for Effect of SH-Wave on Shallow Fill Multiple Circular Cavities. Journal of Harbin Engineering University, 24, 108-113.

[3] Wang, Y. and Liu, D.K. (2002) Dynamic Analysis for Shallow-Embedded Lining Structure Impacted by Incident SH-Wave. Journal of Harbin Engineering University, 23, 43-47.

[4] Lin, H. and Liu, D.K. (2002) Scattering of SH-Wave around a Circular in Half Space. Earthquake Engineering and Engineering Vibration, 22, 9-16.

[5] Chen, Z.G. and Liu, D.K. (2004) Dynamic Response on a Shallowly Cavity of Arbitrary Shape Impacted by Vertical SH-Wave. Earthquake Engineering and Engineering Vibration, 24, 32-36.

[6] Shi, W.P. and Li, C.H. (2012) Scattering of Circular Cavity in Half Space to the Steady Incident Plane SH-Wave. Journal of Mechanical Strength, 34, 86-91.

[7] Wang, Q. and Shi, W.P. (2013) Stimulating Effect of Half-Space Surface Out-of-Plane Line Harmonious Oscillation to Surface Rigid Body. Journal of Mechanical Strength, 35, 438-441.

[8] Shi, W.P., Li, L., Zhang, C.P. and Li, L.C. (2009) Scattering of SH-Wave Circular Cavity in the Half-Space with Elastic Constraint Acting on the Straight Boundary. Journal of Mechanical Strength, 31, 740-746.

[9] Lee, V.W. and Trifunac, M.D. (1979) Response of Tunnels to Incident SH-Wave. Journal of the Engineering Mechanics Division, 105, 643-659.

[10] Manoogian, M.E. and Lee, V.W. (1996) Diffraction of SH-Waves by Subsurface Inclusions of Arbitrary Shape. Journal of Engineering Mechanics, 122, 123-129. https://doi.org/10.1061/(ASCE)0733-9399(1996)122:2(123)

[11] Yuan, X.M. (1996) Influence of Plane Scattering on the Surface Motion of Circular Plugs under the Surface. Journal of Geophysics, 39, 373-381.

[12] Hrachya, B. and Matevosyan, H.H. (2011) Scattering and Transformation of Waves on Heavy Particles in Magnetized Plasma. Journal of Modern Physics, 2, 162-173. https://doi.org/10.4236/jmp.2011.23025

[13] Raju, C.C. (2015) Neutron-Nucleus Total Elastic Scattering. Journal of Modern Physics, 6, 22-25. https://doi.org/10.4236/jmp.2015.61004

[14] Donald, C. (2016) Why Energy and Mass Can Be Converted between Each Other? Anew Perspective Based on a Matter Wave Model. Journal of Modern Physics, 7, 395-403. https://doi.org/10.4236/jmp.2016.74040

[15] Hui, Q., Chen, H., Zhang, X., et al. (2018) Scattering of SH-Wave by an Elliptical Inclusion with Partial Debonding Curve in Half-Space. Waves in Random and Complex Media, 10, 1-18.

[16] Zhou, C.P., Wang, Q.Y., Chen, D., et al. (2018) Elastic Wave Scattering and Dynamic Stress Concentrations in Stretching Thick Plates with Two Cutouts by Using 
the Refined Dynamic Theory. Acta Mechanica Solida Sinica, 3, 1-7. https://doi.org/10.4236/jmp.2016.74040

[17] Gurtin, M.E. and Murdoch, A.I. (1975) A Continuum Theory of Elastic Materials Surfaces. Archive for Rational Mechanics and Analysis, 57, 291-323. https://doi.org/10.1007/BF00261375

[18] Gurtin, M.E. and Murdoch, A.I. (1978) Surface Stress in Solids. International Journal of Solids and Structures, 14, 431-440. https://doi.org/10.1016/0020-7683(78)90008-2

[19] Sharma, P. and Ganti, S. (2004) Size-Dependent Eshelby's Tensor for Embedded Nano-Inclusions in Corporating Surface Energies. Journal of Applied Mechanics, 75, 663-671. https://doi.org/10.1115/1.1781177

[20] Wang, G.F., Wang, T.J. and Feng, X.Q. (2006) Surface Effects on the Diffraction of Plane Compressional Waves by a Nanosized Circular Hole. Applied Physics Letters, 89, 1-3. https://doi.org/10.1063/1.2403899

[21] Ru, Y., Wang, G.F. and Wang, T.J. (2009) Diffractions of Elastic Waves and Stress Concentration Near a Cylindrical Nano-Inclusion Incorporating Surface Effect. Vibration and Acoustics, 131, 1-7. https://doi.org/10.1115/1.4000479

[22] Shen, H. and Schiavone, P. (2000) An Elliptic Inclusion with Imperfect Interface in Anti-Plane Shear. International Journal of Solids and Structures, 37, 4557-4575. https://doi.org/10.1016/S0020-7683(99)00174-2

[23] Wu, H.M. and Ou, Z.Y. (2013) Effect of Surface Elasticity on Scattering of Plane P-Waves by an Elastic Half-Plane with a Semi-Cylindrical Cavity. Applied Mechanics and Materials, 303, 2656-2660. https://doi.org/10.4028/www.scientific.net/AMM.303-306.2656

[24] Ou, Z.Y. and Wu, Y.W. (2015) Effect of Surface Stress on Contact Problem of an Elastic Half Plane with a Circular Cavity. Applied Mathematics and Mechanics, 36, 607-615.

[25] Ou, Z.Y. and Lee, D.W. (2012) Effect of Interface Energy on Scattering of Plane Elastic Wave by a Nano-Sized Coated Fiber. Journal of Sound and Vibration, 331, 5623-5643. https://doi.org/10.1016/j.jsv.2012.07.023

[26] Wang, Z.X. and Guo, D.R. (2000) Conspectus on the Special Functions. Peking University Press, Beijing, 385-386. 
Appendix A

$$
\begin{aligned}
& \varepsilon_{n}=E_{n-2}+E_{n-1}+E_{n}+E_{n+1}+E_{n+2} \\
& E_{n-2}=-\frac{k s}{2}\left\{H_{n-2}^{(1)}[k|\omega(\eta)|]\left[\frac{\omega(\eta)}{|\omega(\eta)|}\right]^{n-2} \frac{\eta^{2}\left[\omega^{\prime}(\eta)\right]}{\left|\omega^{\prime}(\eta)\right|}+\psi^{(4)}\right\} \\
& E_{n-1}=H_{n-1}^{(1)}[k|\omega(\eta)|]\left[\frac{\omega(\eta)}{|\omega(\eta)|}\right]^{n-1} F_{1}-\psi^{(3)} F_{2} \\
& E_{n}=-k s\left\{H_{n}^{(1)}[k|\omega(\eta)|]\left[\frac{\omega(\eta)}{|\omega(\eta)|}\right]^{n}+\psi^{(1)}\right\} \\
& E_{n+1}=H_{n+1}^{(1)}[k|\omega(\eta)|]\left[\frac{\omega(\eta)}{|\omega(\eta)|}\right]^{n+1} F_{2}-\psi^{(2)} F_{1} \\
& E_{n+2}=-\frac{k s}{2}\left\{H_{n+2}^{(1)}[k|\omega(\eta)|]\left[\frac{\omega(\eta)}{|\omega(\eta)|}\right]^{n+2} \frac{\bar{\eta}^{2}\left[\overline{\omega^{\prime}(\eta)}\right]^{2}}{\left|\omega^{\prime}(\eta)\right|}+F_{4}\right\} \text { (A.6) } \\
& F_{1}=\left\{\frac{(1-s) \eta \omega^{\prime}(\eta)-s \eta^{2} \omega^{\prime \prime}(\eta)}{\left|\omega^{\prime}(\eta)\right|}-\frac{s \eta \omega^{\prime}(\eta) \operatorname{Im}\left[\omega^{\prime}(\eta) \bar{\eta} \overline{\omega^{\prime \prime}(\eta)}\right]}{\left|\omega^{\prime}(\eta)\right|^{3}}\right\} \\
& F_{2}=\left\{\frac{(s-1) \bar{\eta} \overline{\omega^{\prime}(\eta)}+s \bar{\eta}^{2} \overline{\omega^{\prime \prime}(\eta)}}{\left|\omega^{\prime}(\eta)\right|}-\frac{s \bar{\eta} \overline{\omega^{\prime}(\eta)} \operatorname{Im}\left[\omega^{\prime}(\eta) \bar{\eta} \overline{\omega^{\prime \prime}(\eta)}\right]}{\left|\omega^{\prime}(\eta)\right|^{3}}\right\} \\
& \psi^{(4)}=\sum_{t=-\infty}^{\infty} \exp \left[\frac{\mathrm{i}}{2}(t-n+2) \pi\right] H_{t-n+2}^{(1)}(2 k h) J_{t}[k|\omega(\eta)|]\left[\frac{\omega(\eta)}{|\omega(\eta)|}\right]^{-t} \frac{\bar{\eta}^{2}\left[\overline{\omega^{\prime}(\eta)}\right]^{2}}{\left|\omega^{\prime}(\eta)\right|} \\
& \psi^{(5)}=\sum_{t=-\infty}^{\infty} \exp \left[\frac{\mathrm{i}}{2}(t-n-2) \pi\right] H_{t-n-2}^{(1)}(2 k h) J_{t}[k|\omega(\eta)|]\left[\frac{\omega(\eta)}{|\omega(\eta)|}\right]^{-t} \frac{\eta^{2}\left[\omega^{\prime}(\eta)\right]^{2}}{\left|\omega^{\prime}(\eta)\right|}( \\
& \varepsilon=\exp \left\{-\frac{k}{2}[\omega(\eta)-\mathrm{i} h]+\frac{k}{2}[\omega(\eta)+\mathrm{i} h]\right\}\left\{\frac{2(s-1) \operatorname{Im}\left[\eta \omega^{\prime}(\eta)\right]}{\left|\omega^{\prime}(\eta)\right|}+\right. \\
& \left.\frac{2 s \operatorname{Im}\left[\eta^{2} \omega^{\prime \prime}(\eta)\right]}{\left|\omega^{\prime}(\eta)\right|}+\frac{2 s \operatorname{Im}\left[\omega^{\prime}(\eta) \overline{\omega^{\prime \prime}(\eta)} \bar{\eta}\right] \operatorname{Re}\left[\eta \omega^{\prime}(\eta)\right]}{\left|\omega^{\prime}(\eta)\right|^{3}}-\frac{2 k s \operatorname{Re}\left[\eta \omega^{\prime}(\eta)\right]}{\left|\omega^{\prime}(\eta)\right|}\right\}
\end{aligned}
$$

